\title{
Intelligent Electrical Apparatus Control Simulation System
}

\author{
Shoujin Wang ${ }^{1, a^{*}}$, Zhaoyang $\mathrm{Xu}^{1, \mathrm{~b}}$ and Jiyuan Feng ${ }^{1, \mathrm{c}}$
}

${ }^{1}$ School of Information \& Control engineering, Shenyang Jianzhu University, Shenyang, China

a23917240@qq.com, b905777203@qq.com, c1970881595@qq.com

\section{*The corresponding author}

Keywords: Building energy saving; Intelligent electrical apparatus; Intelligent monitoring; Electrical switch

\begin{abstract}
With the progress and development of computer science and technology, motion capture is increasingly being used in many fields of intelligent surveillance and intelligent electronic device control and other smart appliances. Intelligent electrical apparatus control simulated system is using the camera monitors whether there is a way to control an electrical switch of the automatic control system. In order to facilitate the realization of simulation system, the paper takes light switch control for example. It could control electrical switches in intelligence, in order to achieve the aim of smart building energy saving.
\end{abstract}

\section{Introduction}

Intelligent Monitor, is to through intelligent image recognition tools to analyze integrated intelligent behavior recognition algorithms, is able to identify and judge the behavior of the screen in a scene or vehicle, and alarm user under appropriate conditions. At the same time, it can stay awake 24 hours to operate, removing a lot of spam, control all movement within the scope of monitoring effectively [1]. Intelligent surveillance liberates video surveillance personnel from the tedious operation and helps them management control objectives effectively and precisely.

Intelligent building is the combination of computer technology, communication technology, information technology and construction technology, through the management of building equipment by automated monitoring and information resources to provide a safe, efficient, comfortable, convenient built environment, the role of energy-saving building automation system in the building cannot be underestimated and replaced in case of how does real true implement intelligent building energy consumption[2,3]. It uses computer control and management, reduces management, saving lighting, air conditioning, energy consumption control system, emphasis on high efficiency, low energy consumption, low pollution, under the premise of people oriented to achieve energy saving, environmental protection and sustainable development goals.

Intelligent analog electrical control system is the set of system that is applied in building automation field. Through the camera, Intelligent electrical apparatus control simulation system will automatically identify whether the personnel present in the building, and control within the building electrical switches, to achieve the goals of lower energy consumption and build green buildings.

\section{Design Ideas}

Intelligent electrical apparatus apparatus simulated control system is a set of automatic control system that to use the camera to monitor whether people existing to control electrical switch. The achievement of camera capturing the action information is from introducing a dynamic focus capture technology package library implementation [4]. The range of people entering into camera will be monitored as a large dynamic focus, triggers electrical opening operation; the range of people leaving from the camera will not be detected as a large dynamic focus, triggers electrical opening and closing operations.

Considering there are many types of appliances within the building, such as lighting, air conditioning, elevators, ventilation, etc. [5]. This software system will take simulate lamp for an 
example, is able to control light switch intelligently in order to achieve smart building energy saving.

\section{Functions Partition}

According to the software demands, the system functions can be partitioned as follows:

(1) Launch. Initializing related code and launch the camera.

(2) Exit. Exit the software and release all system resources.

(3) Set the background. Set the background image of the Dynamic Focus Capture System.

(4) Adjustment of threshold value / sensitivity. Adjust the operation conditions to fine-tune recognition.

(5) Monitoring start (end). The captured process of start (end) motion.

(6) Monitoring pause (resume). The captured process of pause (resume) motion.

(7) About. Introduce software developers' information and version information.

The system function structure is shown in Fig. 1.

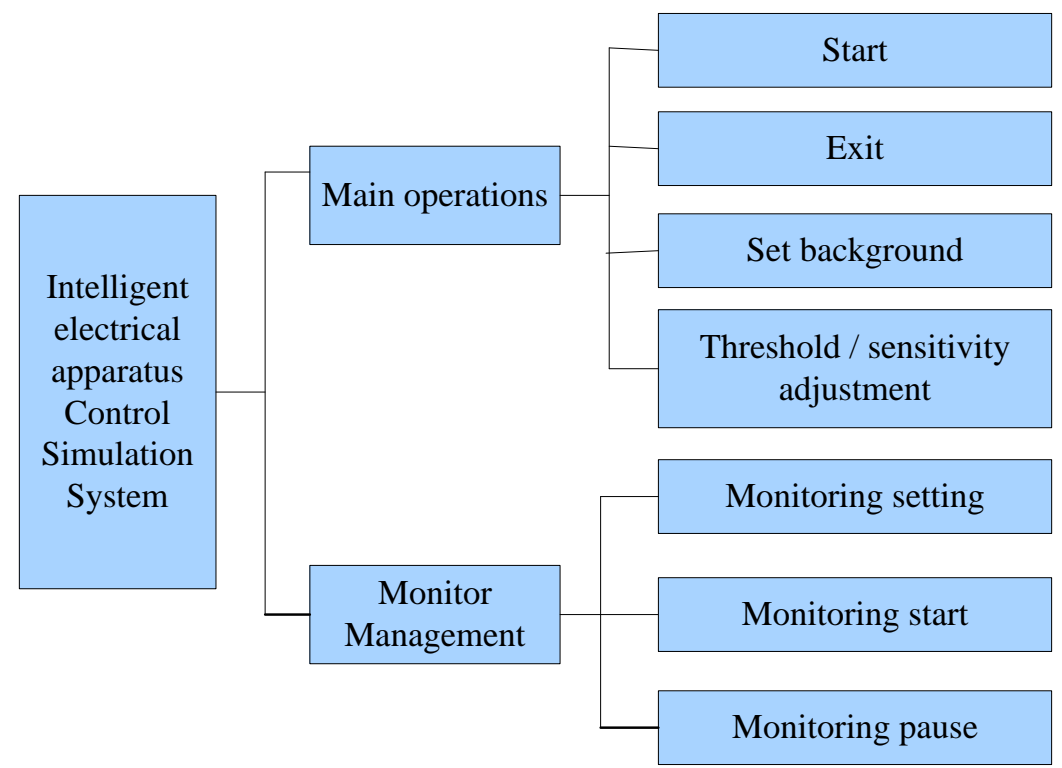

Figure 1. System functions chart

\section{Core Functions Design}

Function Introduction. This set of core functionality of the system is determined within the range of the camera there were no activities. If monitoring person activity, turn on the corresponding electrical appliances; otherwise, turn off the corresponding electrical appliances [6]. The determined motion can be achieved by the dynamic motion capture technology focus in the package library implemented method. It can monitor the dynamic range of the camera in focus. If there is a dynamic focus greater than a certain threshold, it can be determined that there is staff activity .

Detection process will use independent threads running loops for some code to implement monitoring. Control appliances belong to the external interface. This article will simulate lamp effect instead.

Detailed Design. Input: Justify threshold and thread control command.

Algorithm description: Pseudo code of the core functions is described as follows:

Cyclic execution of the code segment

Thread waiting for 1 second

Enter the current frame for the frame to be calculated

Computational dynamic focus

If (the focus is greater than the threshold) 


\section{\{Turn on the light $\}$ \\ OR \\ \{Turn off the light $\}$}

Output: the status of light switch

\section{Threshold Centroid Algorithm}

Overview of Threshold Centroid Algorithm. Firstly, thresholding the image and then use COG algorithm. Essence threshold centroid algorithm can refer to the following formula (1), limited to a fixed threshold, the original binary image [7, 8]. Using the weighted average of the idea in the horizontal and vertical directions, find the corresponding position of the particle respectively. This algorithm is to replace the late herein iterative centroid algorithm.

$$
\begin{gathered}
x=\frac{\sum_{i}^{m} \sum_{j}^{n} i I(i, j)}{\sum_{i}^{m} \sum_{j}^{n} I(i, j)}, \quad y=\frac{\sum_{i}^{m} \sum_{j}^{n} j I(i, j)}{\sum_{i}^{m} \sum_{j}^{n} I(i, j)} \\
I(\boldsymbol{i}, j)= \begin{cases}1, & (i, j) \in \text { target } \\
0, & (i, j) \in \text { background }\end{cases}
\end{gathered}
$$

Description Threshold Centroid Algorithm. This paper will have a further description of centroid algorithm iteratively in the form of pseudo-code.

Focus function (Rectangular area rec, Target contrast gray value target)

Arr after array zero

For $(\mathrm{i}=0 ; \mathrm{i}<$ image width; $\mathrm{i}++)$

\{

For $(\mathrm{j}=0 ; \mathrm{j}<$ Picture height $; \mathrm{j}++)$

\{

if (point $(i, j)$ in Rectangular area rec)

\{

If ((The difference between the pixel and the background image is

larger than the threshold value.)

And (The pixel point is near the target gray value.))

ey add j;

$$
\text { Ex Add i; }
$$

Particle mass accumulation1;

Else

\{

Current pixel setting 0;

$$
\text { \} }
$$$$
\}
$$

\}

Focal transverse coordinates=ex/quality

Focal longitudinal coordinates=ey/quality;

Update focus information; 
Study on Performance Thresholds Centroid Algorithm. Set the image width w, height h, $\mathrm{n}$ detected valid pixel, then the main computing amount when obtaining its main focus in the process is:

$\mathrm{N}$ the second process is $\mathrm{A}$, in which the process $\mathrm{A}$ is expressed as

Process A \{

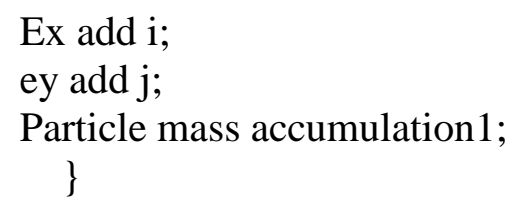

\}

Suppose process A cost time Ta.

From the above analysis, it can be acquired that the entire calculation process mainly takes $\mathrm{T}=\mathrm{n}$ * Ta. Time complexity threshold centroid algorithm is $\mathrm{O}(\mathrm{n})$.

The whole process has been calculated using a two-dimensional array to store the binary image data, so the space threshold centroid algorithm complexity is $\mathrm{O}(\mathrm{w} * \mathrm{~h})$.

Compilation Tools system for the Microsoft Visual Studio, target frame for .NetFramewoke 3.5, the assembly name is AutoTurnOn, output type is a Windows application, the main executable file AutoTurnOn.exe. After opening the main interface, open the camera effect is shown in Fig. 2.

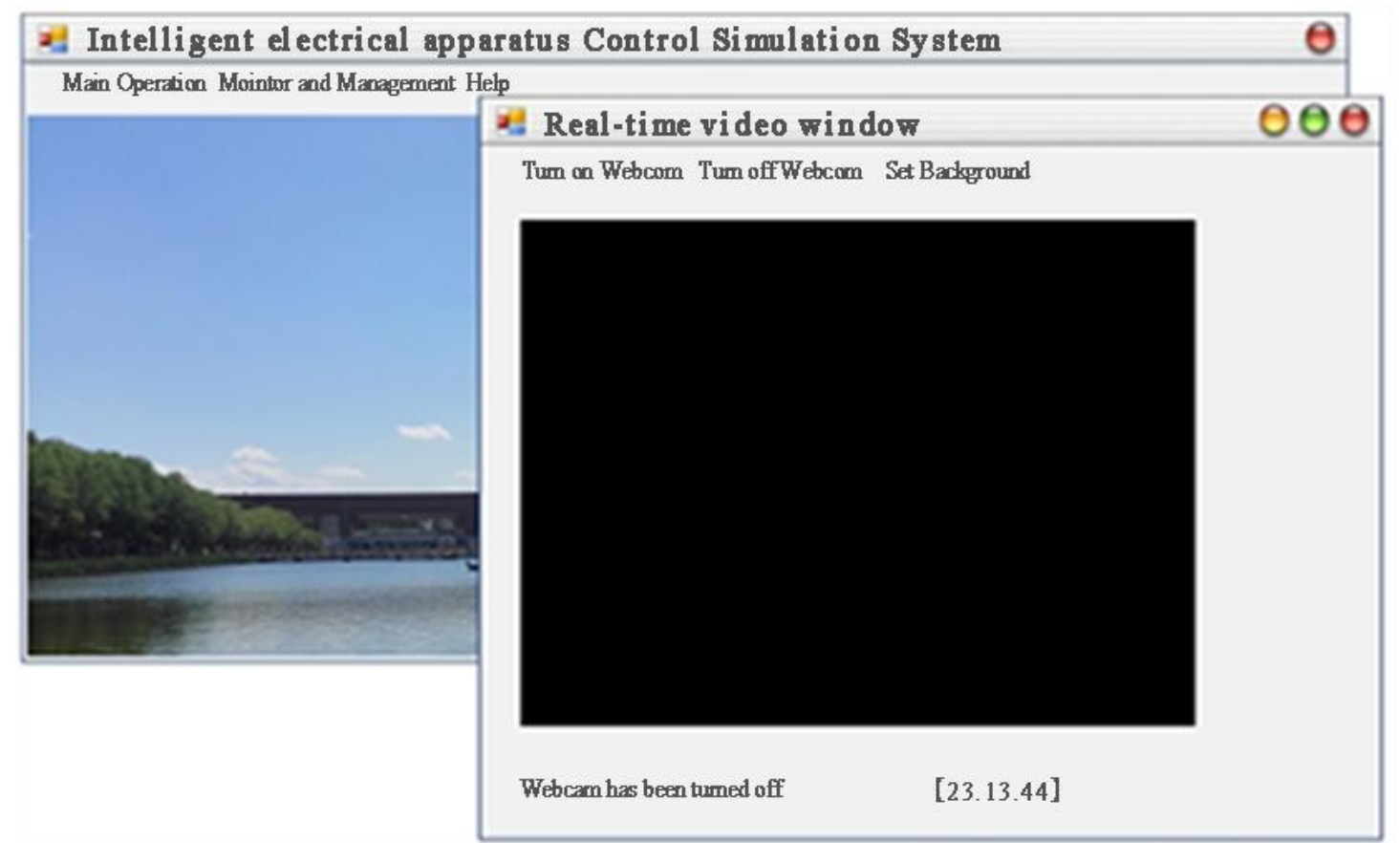

Figure 2. The main interface and the camera live window

Set the background, after the start of testing, the current non-entry into camera range, the lamp is not switched on. Specific results shown in Fig. 3. 


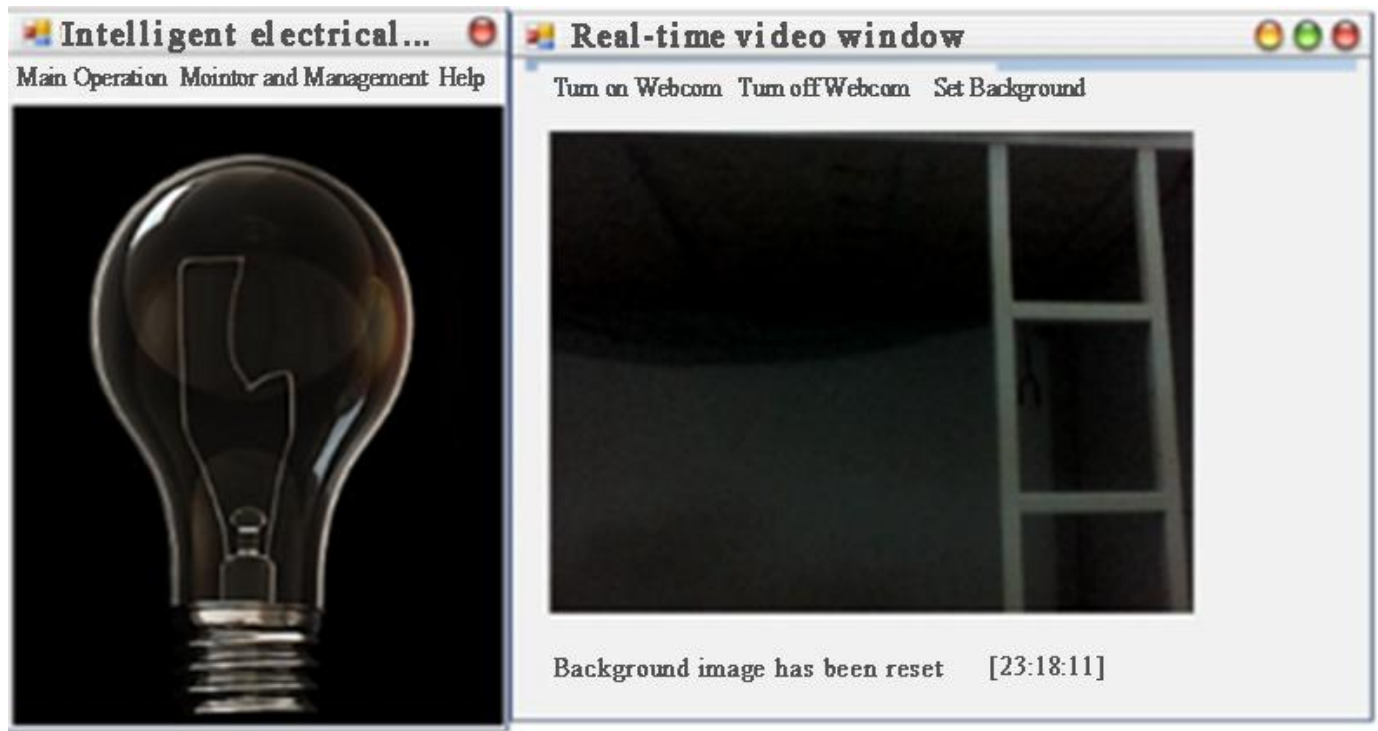

Figure 3. Shortcut of monitor process (No people entered)

When people entered the camera range, the system successfully detected personnel and switched light. Specific results shown in Fig. 4.

Testing results: Through testing, this system could work properly. It could determine sensitively, control electrical precisely and achieve the desired objectives well.

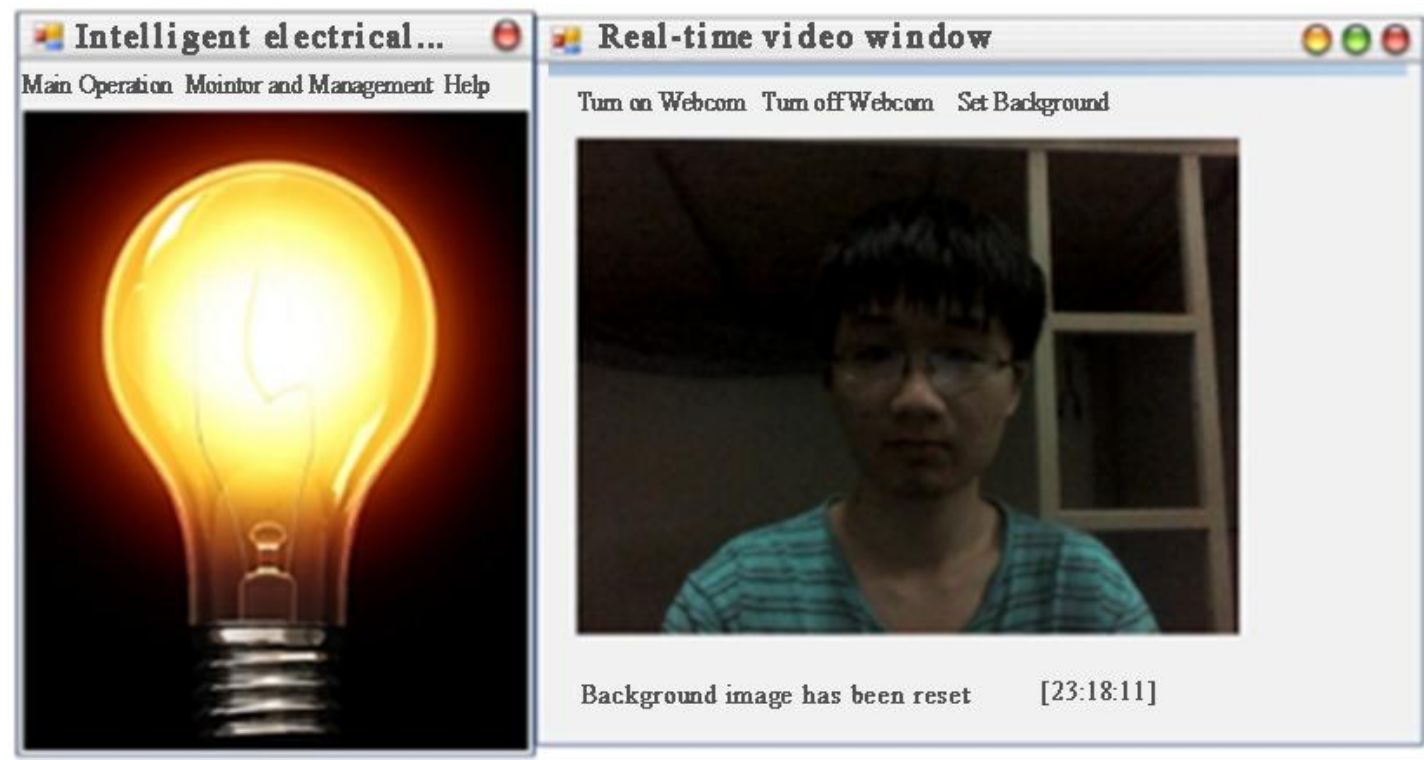

Figure 4. Shortcut of monitor process (People entered)

\section{Conclusions}

This paper studies how to base on single camera-based hardware, to achieve the detection and capture of the target moving object or motion. System input device used in an ordinary camera that can capture images and color information. It uses color information through the centroid algorithm, captures the position of the target object and obtains sports focus. It can determine the action to be captured has happened or not by analysing location and range of variation separately over time. Through analyzing and abstracting the focus that this system captured, we can divide the movement to be detected easily, and trigger electrical switches to achieve electrical achieve intelligent control. 


\section{References}

[1] Jiang Mingyan, the video image processing in traffic statistics algorithm [D]. Chengdu: University of Electronic Science and technology, 2013:37 38

[2] Sha youth. Video based traffic statistics algorithm research [D]. Wuhan: Huazhong University of Science and Technology, 2013:44 45

[3] Rahman A, Yella S, Dougherty M. Simulation and Optimization Techniques for Sawmill Yard Operations-A Literature Review [J]. Journal of Intelligent Learning Systems \& Applications, 2014, 06(1):21-34.

[4] Shoujin Wang, Ziyang Han, Song Guo. Design and Implementation on Gesture Recognition Game Controller[C]// 6th International Conference on Electronic, Mechanical, Information and Management Society. 2016.

[5] Shoujin Wang, Yang Cao, Jingang Shi. Dynamic Focus Capture Method and Its Application[C]// 6th International Conference on Electronic, Mechanical, Information and Management Society. 2016.

[6] Wang Z, Si J F, Hui L S. Design of Intelligent Actuation Time Measurement for Low Voltage Electrical Apparatus [J]. Advanced Materials Research, 2013, 709:379-382.

[7] Li Zhaokun. Human computer interaction system based on motion recognition technology [D]. Suzhou: Soochow University, 2014:12 17

[8] Min Lin, Bin Li, Qiao -Hong Liu. Identification of Eye Movements from Non-front--al Face Images for Eye-controlled Systems[J]International Journal of Automation \& C-omputing,2014,issue No.5: 29 30 